\title{
Homeopathic Viscum Album on the Treatment of Scamous Cell Carcinoma Lesion in a Dog (Canis familiaris) - Case Report
}

\author{
Ana Catarina Viana Valle ${ }^{1,2 *}$ and Aloisio Cunha de Carvalho ${ }^{1,3}$ \\ ${ }^{1}$ Doctor Izao Soares Institute, Integrative Medicine, Ribeirao Preto, SP, Brazil \\ ${ }^{2} \mathrm{PhD}$ in Genetics and Biotechnology, Catholic University, Brazil \\ ${ }^{3} \mathrm{PhD}$ in Ambiental Pathology, Paulista University, Brazil
}

${ }^{*}$ Corresponding author: Ana Catarina Viana Valle, Doctor Izao Soares Institute, Integrative Medicine, Ribeirao Preto, SP, PhD in Genetics and Biotechnology, Catholic University, Brazil; E-mail: dranacatarina@gmail.com

Received: May 02, 2021; Accepted: May 09, 2021; Published: May 15, 2021

\begin{abstract}
Squamous Cell Carcinoma (SCC) is characterized by the uncontrolled growth of abnormal skin cells. The disease affects dogs and cats generally with advanced age and has a high incidence in white and/or depigmented animals. In some situations, the conventional treatments do not contemplate the cure nor the improvement of life quality of the patients affected by this disease. For this reason, the Viscum album therapy has been indicated for treating cancer patients when conventional medicine is not an option to be considered anymore, as well in Palliative Care aiming to maintain the patient's quality of life. This study aimed to report the healing process of a difficult resolution lesion, due to a squamous cell carcinoma, in a 9-year-old dog (Canis familiaris), PitBull breed. The disease was treated by the intravenous Viscum album therapy associated with Hamamellis virginiana, which was orally administered. The patient showed excellent response to the prescribed treatment with complete healing of a lesion derived from a SCC in only four weeks. Improvement in appetite and overall disposition were also recorded.
\end{abstract}

Keywords: Squamous cell carcinoma, Hamamellis virginiana, Viscum album, Therapy

\section{Introduction}

Squamous cell carcinoma (SCC) is a malignant neoplasia relatively common in dogs and cats [1]. The disease development is directly related to skin exposition to UV rays. However, it can also be related to burnings, previous non-malignant lesions, and chronic inflammatory diseases [2]. SCC has a higher incidence in dogs with advanced age among 6-10 years. There is no sex predisposition. The breeds Collie, Basset Hound, Schnauzer, Dalmatian, Pitbull, and Beagle seem to have a higher risk for developing this disease. Dogs of white and short fur with white or spotted ventral body parts also seem to be predisposed [2]. Diagnosis occurs by histopathological analysis of the injured tissue. The treatment of choice is by surgical resection, when possible, intralesional chemotherapy, phototherapy, cryosurgery, and electrotherapy according to the localization, evolution time, and progression of the disease [2]. Nevertheless, all treatments considered by conventional medicine cause moderate to severe side effects to the patients. Also, many times, they are not efficient. Complementary therapies have gained notoriety in cancer treatment either as a single or complementary therapy. Within this context, the Viscum album therapy is the complementary treatment most indicated by the medical doctors in Germany and Switzerland for treating cancer patients. One of the main benefits of this medicine is its selective toxicity activity on tissues affected by cancer. Viscum album presents a bidirectional activity for the patients since it can also immunomodulate the host organism [3].

Therefore, this study aimed to report the healing process of a lesion of difficult resolution due to a squamous cell carcinoma in a 9-year-old dog, PitBull breed.

\section{Material and Methods}

A 9-year-old female PitBull with white fur, $29 \mathrm{Kg}$, fed with commercial dog food, was seen at NaturalPet Clinic in Brasilia, DF, Brazil. The patient was referred from another colleague with the main complaint of a difficult to heal lesion in the abdomen and the impossibility of its surgical removal (Figure 1). The previous diagnosis was squamous cell carcinoma. The lesion had already been treated by conventional chemotherapy but with no success. On physical examination, the animal was alert to stimuli, had normal mucous membranes, and CRT 2". The cardiac auscultation and cardiac and respiratory frequencies were within the normal range considering age and species. The patient was overweight, in normal hydration conditions, and had opaque and dry fur. Blood was collected for complete blood count and biochemical measurements. The treatment was initiated on the same day using the Viscum album therapy. One

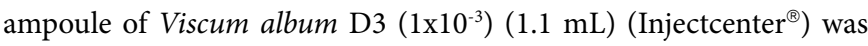
intravenously administered. Additionally, Hamamellis virginiana 


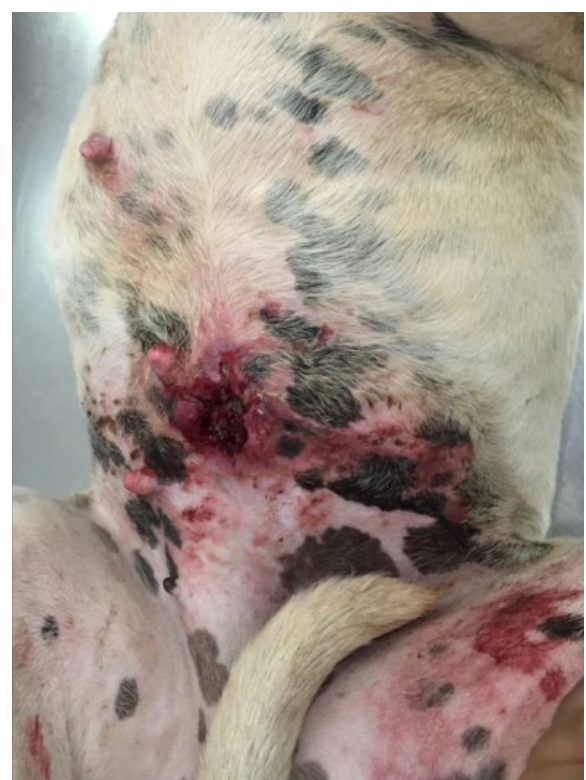

Figure 1: Overall abdomen appearance of the animal with an ulcerated and difficult to heal abdominal lesion.
$30 \mathrm{CH}\left(1 \times 10^{-60}\right)$, three drops, SID, for 30 days; and Omega $32000 \mathrm{mg}$, SID, for 60 days, were orally prescribed. It was recommended for the animal to begin a low-carb diet containing $10 \%$ carbohydrate. The tutor was also advised that the animal should come back at a 7-day interval for Viscum album D3 intravenously applications for 60 days.

\section{Results}

The result of the blood tests showed: Red blood cells - 7,750,000/ uL; Hemoglobin - 17.7 g/dL; Hematcrit - 53.5\%; Leukocytes - 7,600/ mL; Eosinophils - 152/mL; Lymphocytes - 1,444/mL; Platelets 324,000/mL; Albumin - $3.26 \mathrm{~g} / \mathrm{dL}$; Alanine aminotransferase - $57 \mathrm{U} / \mathrm{L}$; Aspartate aminotransferase - $87 \mathrm{U} / \mathrm{L}$; Creatinine - $1.23 \mathrm{mg} / \mathrm{dL}$; Alkaline phosphatase - $29 \mathrm{U} / \mathrm{L}$; Total proteins - $7.14 \mathrm{~g} / \mathrm{dL}$; and Urea - $29 \mathrm{mg} / \mathrm{dL}$.

Seven days after treatment initiation, the patient returned for the endovenous Viscum album administration, and the lesion already had another appearance. The edema was smaller than the observed in the first evaluation, and so was the lesion size (Figure 2A). The progressive improvement was observed every seven days throughout four weeks (Figures 2B-D).
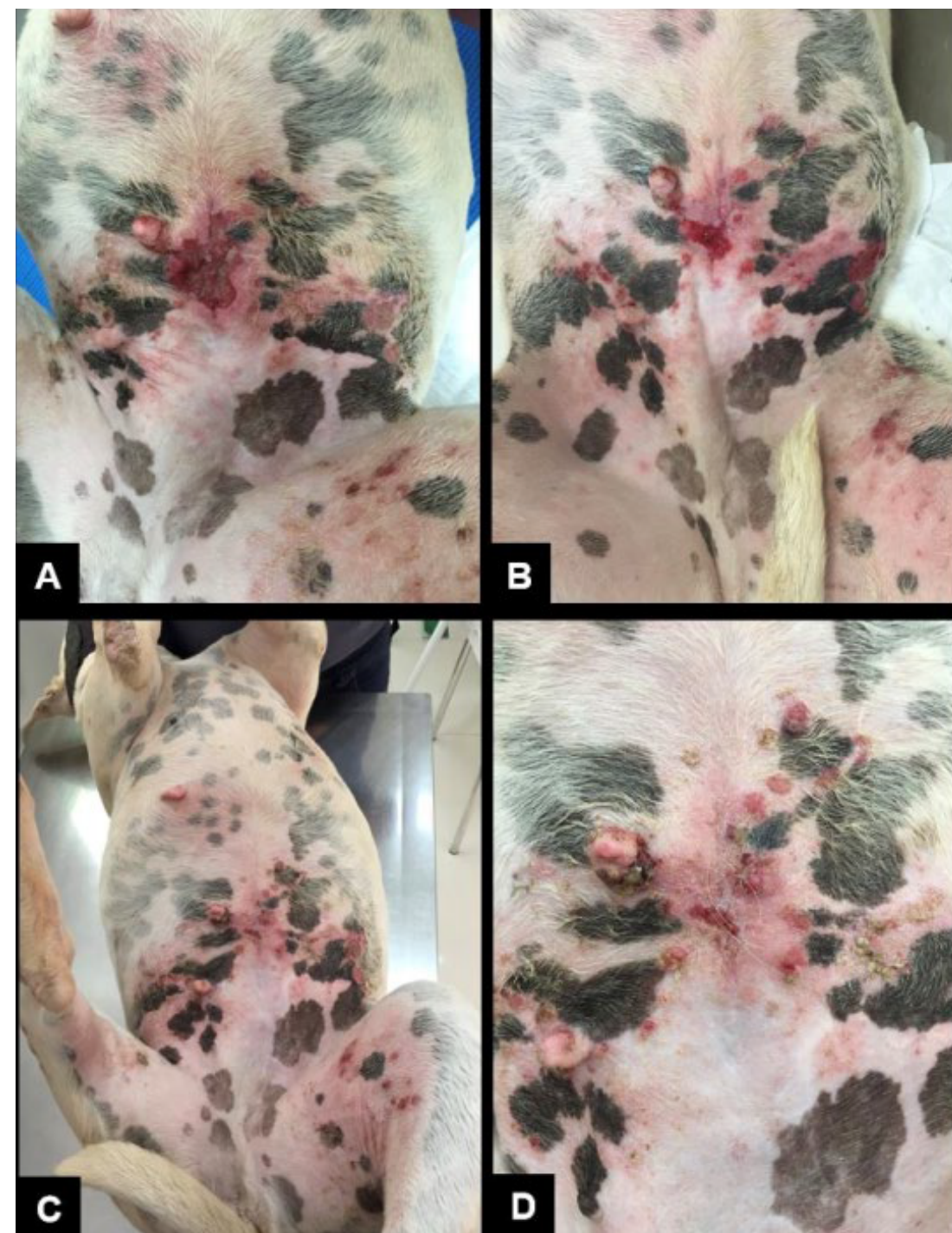

Figure 2: Lesion appearance seven (A), 14 (B), and 21 (C) and (D) days after treatment initiation. 
The tutor reported that the animal was more cheerful than before previously, and its appetite had improved. It also restarted playing with the other dogs of the house, which has not happened for months. This patient was followed up by the total healing period of the lesion. After the lesion has healed, the owner did not want to continue the treatment. Six months after the lesion has healed, another colleague took care of the animal, within the principles of conventional medicine. The complaint was claudication in the left posterior limb. A new investigation was initiated, and osteosarcoma was diagnosed. The patient died in 30 days by euthanasia.

\section{Discussion}

Squamous Cell Carcinoma is characterized by the uncontrolled growth of abnormal cells that appear in the squamous cell layer of the epidermis [1]. The conventional treatment protocols include surgical intervention, chemotherapy, and radiation, which possess various adverse effects [4].

Many homeopathic and phytotherapeutic medicines (Complementary Medicine) with anticancer properties, similar to the conventional medicines for treating cancer, are currently available [4], such as Viscum album. The use of complementary therapies is common among patients with advanced cancer. The role of the therapies used in Palliative Care is to guarantee the best quality of life for patients to whom healing is no longer an option. On the other hand, comfort and welfare must be reached as much as possible until the moment of death.

Within this context, Kienle and Kiene [5] had already recorded in a systematic review the best quality of life of patients using the Viscum album therapy, especially in cases of advanced cancer. Similarly, Valle and Carvalho [6] reported enhanced life quality, including improved overall condition, appetite, and activity in a patient with cutaneous melanoma under Palliative Care. Our findings are corroborated by these authors and described a clear improvement in the patient's quality of life, which was verified not only for the lesion clinical improvement but also for enhancing the animal's disposition and appetite. The animal started to do activities that she did not use to do for a long time, such as playing and inviting other dogs to play. She also became more active, less sleepy, and less tired.

In this study, the animal was systematically treated for approximately one month until lesion healing and had another six months of survival until the osteosarcoma diagnosis in the posterior limb. Its tutor opted for euthanasia. Among the various roles of the Viscum album therapy for the cure and treatment of cancer patients, it plays a critical action within Palliative Care. Under this perspective, this therapy aims to maintain the quality of life of patients in cases when the cure of the disease is no longer possible, but only the disease control, providing welfare until the moment of the death.

\section{Conclusion}

The Viscum album therapy associated with Hamamellis virginiana proved to be efficient in its purpose of healing an ulcerated lesion derived from the SCC development. This therapy also improved the patient's life quality and clearly showed that Integrative therapies could be intrinsic to Palliative Care. Additional studies must be developed for the confirmation of such effects.

\section{References}

1. Ciani F, Tafuri S, Troiano A, Cimmino A, Fioretto BS, et al. (2018) Anti- proliferative and pro-apoptotic effects of Uncaria tomentosa aqueous extract in squamous carcinoma cells. J Ethnopharmacol 211: 285-294. [crossref]

2. Daleck CR, De Nardi AB (2017) Oncology in dogs and cats. $2^{\text {nd }}$ ed, Rocca, Rio de Janeiro. 343p.

3. Valle ACV (2020) In vitro and in vivo evaluation of the ultra-diluted Viscum album efficacy and safety. Doctorate dissertation. Catholic University of Brasilia - UCB, Brasilia-DF, Brazil. 78p.

4. Magadi VP, Ravi V, Arpitha A, Litha, Kumaraswamy K, Manjunath K (2015) Evaluation of cytotoxicity of aqueous extract of Graviola leaves on squamous cell carcinoma cell- 25 cell lines by 3-(4,5-dimethylthiazol-2-Yl) -2,5-diphenyltetrazolium bromide assay and determination of percentage of cell inhibition at G2M phase of cell cycle by flow cytometry: An in vitro study. Contemp Clin Dent 6: 529-533. [crossref]

5. Kienle GS, Kiene H (2010) Review article: Influence of Viscum album L (European mistletoe) extracts on quality of life in cancer patients: a systematic review of controlled clinical studies. Integr Cancer Ther 9: 142-157. [crossref]

6. Valle ACV, Carvalho AC (2021) Ultra-diluted Viscum album in the treatment of cutaneous melanoma in a dog (Canis familiaris) - Case report. Paripex Indian J Res 10: 1-4.

\section{Citation:}

Ana Catarina V. Valle and Aloísio C. Carvalho (2021) Homeopathic Viscum album on the treatment of Scamous Cell Carcinoma Lesion in a Dog (Canis familiaris) - Case Report. Integr J Vet Biosci Volume 5(2): 1-3. 